\title{
The influence of magnetocrystalline anisotropy on the magnetocaloric effect: A case study on $\mathrm{CO}_{2} \mathrm{~B}$ \\ M. Fries, K. P. Skokov, D. Yu. Karpenkov, V. Franco, S. Ener, and O. Gutfleisch
}

Citation: Appl. Phys. Lett. 109, 232406 (2016); doi: 10.1063/1.4971839

View online: http://dx.doi.org/10.1063/1.4971839

View Table of Contents: http://aip.scitation.org/toc/apl/109/23

Published by the American Institute of Physics

\section{Articles you may be interested in}

Heterogeneous nucleation and heat flux avalanches in $\mathrm{La}(\mathrm{Fe}, \mathrm{Si}) 13$ magnetocaloric compounds near the critical point

Appl. Phys. Lett. 109, 231904231904 (2016); 10.1063/1.4971360

Discovery of ferromagnetism with large magnetic anisotropy in ZrMnP and HfMnP

Appl. Phys. Lett. 109, 092402092402 (2016); 10.1063/1.4961933

Adiabatic magnetocaloric effect in Ni50Mn35In15 ribbons

Appl. Phys. Lett. 109, 212402212402 (2016); 10.1063/1.4968592

Ruddlesden-Popper interface in correlated manganite heterostructures induces magnetic decoupling and dead layer reduction

Appl. Phys. Lett. 109, 232405232405 (2016); 10.1063/1.4971833

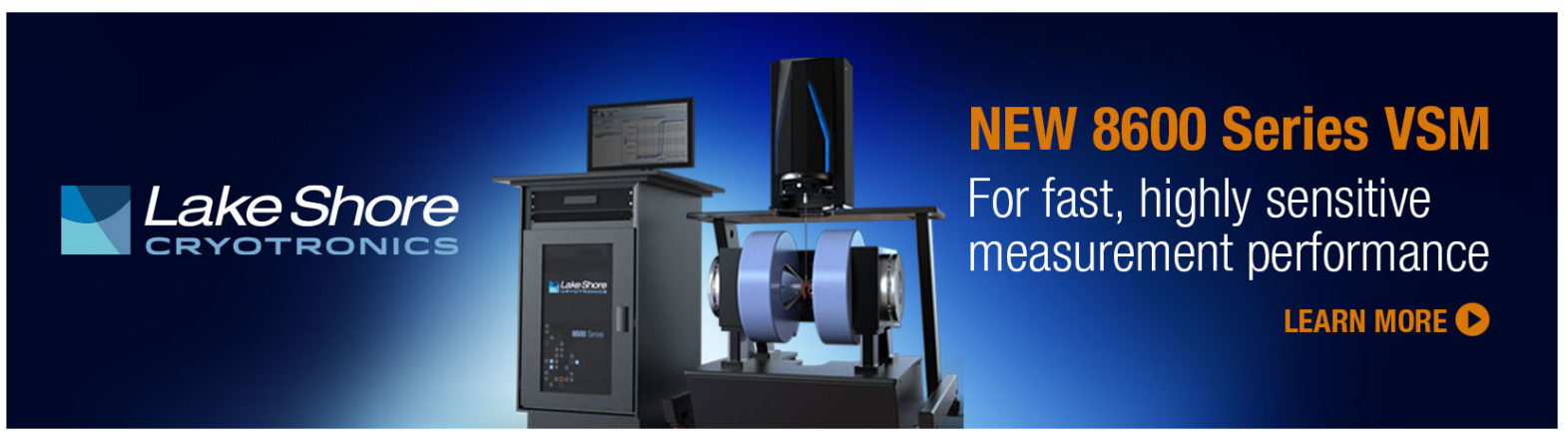




\title{
The influence of magnetocrystalline anisotropy on the magnetocaloric effect: A case study on $\mathrm{CO}_{2} \mathrm{~B}$
}

\author{
M. Fries, ${ }^{1, a)}$ K. P. Skokov, ${ }^{1}$ D. Yu. Karpenkov, ${ }^{1,2}$ V. Franco, ${ }^{3}$ S. Ener, ${ }^{1}$ and O. Gutfleisch ${ }^{1, b)}$ \\ ${ }^{1}$ Institut für Materialwissenschaft, TU Darmstadt, Alarich-Weiss-Str.16, 64287 Darmstadt, Germany \\ ${ }^{2}$ NUST “MISiS," Leninskiy pr.4, 119049, Moscow, Russia \\ ${ }^{3}$ Dpto. Fsica de la Materia Condensada, ICMSE-CSIC, Universidad de Sevilla, P.O. Box 1065, 41080-Sevilla, \\ Spain
}

(Received 28 October 2016; accepted 25 November 2016; published online 6 December 2016)

\begin{abstract}
The influence of magnetocrystalline anisotropy on the magnetocaloric effect (MCE) was studied on single crystals of $\mathrm{Co}_{2} \mathrm{~B}$ and compared to measurements on polycrystalline samples. Large differences in adiabatic temperature change $\Delta T_{a d}$ and magnetic entropy change $\Delta S_{M}$ were found along the different crystallographic directions. The magnetocaloric effect differs by $40 \%$ in the case of $\Delta T_{a d}$ in a field change of $1.9 \mathrm{~T}$ when applying the field along the hard axis and easy plane of magnetization. In the case of $\Delta S_{M}$, the values differ $50 \%$ and $35 \%$ from each other in field changes of 1 and $1.9 \mathrm{~T}$, respectively. It was found that this anisotropy effect does not saturate in fields up to $4 \mathrm{~T}$, which is higher than the anisotropy field of $\mathrm{Co}_{2} \mathrm{~B}(\approx 2 \mathrm{~T})$. A simple model was developed to illustrate the possible effect on magnetocrystalline anisotropy, showing large differences especially in application relevant fields of about $1 \mathrm{~T}$. The results strongly suggest that the MCE could be maximized when orienting single crystalline powders in an easy axis parallel to the applied field in active magnetocaloric regenerator structures, and therefore the overall device efficiency could be increased. Published by AIP Publishing. [http://dx.doi.org/10.1063/1.4971839]
\end{abstract}

Due to its higher theoretical energy efficiency, magnetic refrigeration is considered a promising alternative to conventional gas compression cooling. ${ }^{1,2}$ Since the discovery of the giant magnetocaloric effect in $\mathrm{Gd}_{5}\left(\mathrm{Si}_{2} \mathrm{Ge}_{2}\right),{ }^{3}$ many promising material families like La-Fe-Si- ${ }^{4-7} \quad \mathrm{Fe}_{2} \mathrm{P}$-based alloys, ${ }^{8-10}$ and Heusler alloys ${ }^{11-13}$ have been extensively studied. In these materials, the transition temperatures are highly tunable by utilizing various elemental substitutions or external fields. Most studies on magnetocaloric compounds have been conducted using isotropic polycrystalline samples considering only the conventional magnetocaloric effect, peaking at the Curie temperature. This can be explained by the fact that most magnetocaloric compounds are magnetically soft and are of cubic crystal structure ${ }^{14,15}$ and therefore the anisotropic contribution at the Curie temperature is non existent.

Yet it has been found that especially the $\mathrm{Fe}_{2} \mathrm{P}$-type materials that are industrially viable ${ }^{16}$ show large magnetocrystalline anisotropy, ${ }^{17}$ which could strongly influence the magnetocaloric properties. ${ }^{18}$ In order to build an efficient cooling device, the magnetocaloric effect, namely, the adiabatic temperature change $\Delta T_{a d}$ and isothermal entropy change $\Delta S$, needs to be maximized within the limitations of the available field source. Therefore, the effect of anisotropy is not only of theoretical importance but also of practical significance when designing a heat exchanger made of a magnetocaloric material. Yet till now detailed experimental studies on the effect of anisotropy on the magnetocaloric effect are missing because the analysis of anisotropic effects is

\footnotetext{
a)fries@fm.tu-darmstadt.de

b) gutfleisch@fm.tu-darmstadt.de
}

generally hindered by the lack of single crystal data on magnetocaloric materials.

The anisotropy of the magnetocaloric effect (MCE) has already been discovered several decades ago. Vonsovskiy theoretically predicted a difference in heat capacity at a different magnetic field orientation for a cobalt single crystal in $1938 .{ }^{19}$ In the work of Akulov and Kirensky from $1940,{ }^{20}$ the rotational MCE was directly measured on a nickel single crystal. According to these works, the anisotropic effect in pure $3 \mathrm{~d}$ metals is below $0.1 \mathrm{~K} / \mathrm{T}$ due to low values of their magnetocrystalline anisotropy. However, it was found that the MCE in single crystals of rare earth (RE) metals can be strongly anisotropic, with a different sign of MCE when applying a field along easy and hard axes of a single crystal. ${ }^{21,22}$

According to recent studies conducted on single crystals, the magnitude and shape of the magnetic entropy change can be significantly different when the external field is applied along different crystallographic directions. ${ }^{23-31}$ However, the temperatures at which this effect was observed lie far below room temperature.

In this letter, we report on the effect of magnetocrystalline anisotropy near the Curie temperature and its influence on the magnetocaloric effect. We chose a single crystal of $\mathrm{Co}_{2} \mathrm{~B}$ as for reference as this system offers a well-defined determination of magnetocrystalline anisotropy. The magnetic properties of $\mathrm{Co}_{2} \mathrm{~B}$ were reported in Ref. 32 both for theoretical and experimental aspects. It was found that the anisotropy changes from an easy axis anisotropy [001] to an easy plane anisotropy (100) spanned by the axis [100] and [010] with a spin reorientation at around $78 \mathrm{~K}$ with an anisotropy field $H_{A}$ of approximately $2 \mathrm{~T}$. The Curie temperature $T_{C}$ of the alloy is $420 \mathrm{~K}$. The crystal lattice of $\mathrm{Co}_{2} \mathrm{~B}$ with the spins aligned along the [100] direction is depicted 
schematically in the inset of Figure 1. For a typical domain structure of a single crystal with easy plane anisotropy, the reader is referred to Ref. 22.

The $\mathrm{Co}_{2} \mathrm{~B}$ pre-alloys were prepared by induction melting of commercial purity Co $(99.99 \%$, Mateck) and B (99.999\%, Mateck) under argon atmosphere. For obtaining single crystals, the ingots were remelted under Ar atmosphere at $1583 \mathrm{~K}$ and slowly cooled to room temperature with a cooling rate of $10 \mathrm{~K} / \mathrm{h}$ leading to enhanced grain growth. Large single crystalline grains were extracted and the crystalline quality and orientations were checked by Laue and powder X-ray diffraction. For magnetic measurements, the sample was cut into a $1 \times 1 \times 1 \mathrm{~mm}$ cube with edges parallel to [001], [100], and [010]. The polycrystalline sample was taken from the same pre-alloy batch as the single crystal. The size and shape of the polycrystalline sample were chosen to be comparable with the single crystal in order to account for possible size and demagnetization effects. In order to study the influence of magnetocrystalline anisotropy on the magnetocaloric effect, the adiabatic temperature change was obtained by means of direct measurements in a purpose-built setup described in Ref. 33 and magnetic isotherms recorded on a Quantum Design 14 T PPMS (Physical Property Measurement System) magnetometer and analyzed using the Maxwell relation. Both measurements were carried out on the single crystal along [001], [100], and [010] (equivalent) directions and on the polycrystalline sample.

The $\mathrm{Co}_{2} \mathrm{~B}$ sample crystallizes in space group $\mathrm{I} / \mathrm{mcm}$ (\#140) with lattice parameters of $a$ and $b=0.501 \mathrm{~nm}$ and $c=0.422 \mathrm{~nm}$, which is in good agreement with the literature. ${ }^{34}$ The chemical and phase purity of the samples was confirmed by scanning electron microscopy in the BSE (backscattered electron) mode and X-ray diffraction.

The adiabatic temperature change $\Delta T_{a d}$ measured in a field change of $1.9 \mathrm{~T}$ is shown in Figure 1. The maximum adiabatic temperature change for an external field applied along the hard axis [100] is $0.65 \mathrm{~K}$ at a temperature of $425 \mathrm{~K}$. This is around $40 \%$ lower than that compared for applying

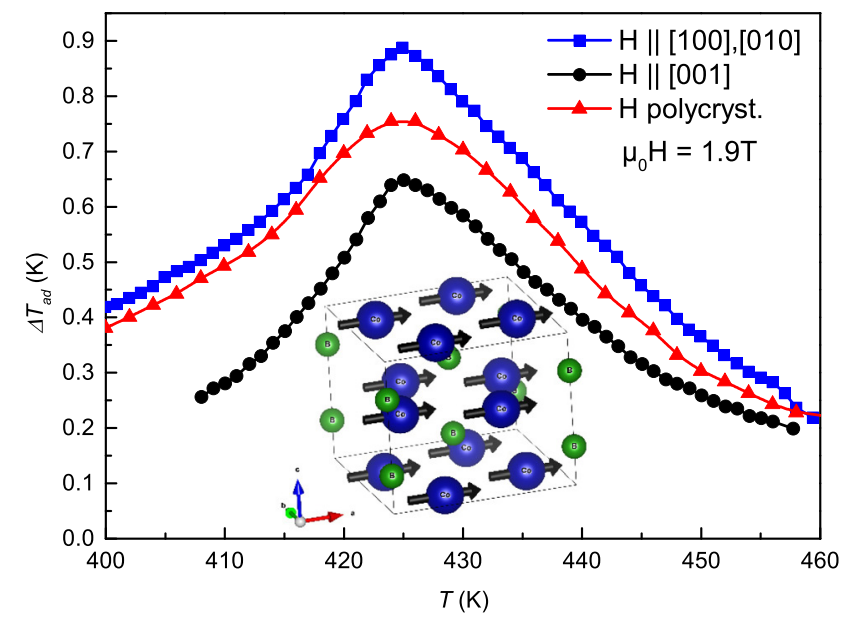

FIG. 1. $\Delta T_{a d}$ versus $\mathrm{T}$ in field changes of 1.9 T measured along [100], [010], and [001] directions shown in blue squares and black dots. Measurements performed on a polycrystalline samples are shown with red triangles. The inset shows a schematic of the crystal lattice of $\mathrm{Co}_{2} \mathrm{~B}$ with the spins aligned along the [100] direction. the field in the easy plane $(100)$, which is $0.90 \mathrm{~K}$. The polycrystalline measurements lie in between the values obtained for applying a field along the easy plane and hard direction of magnetization. A maximum adiabatic temperature change of $0.75 \mathrm{~K}$ is observed for the polycrystalline sample. In all cases, the peak $\Delta T_{a d}$ takes place at the same temperature of $425 \mathrm{~K}$.

The field dependence of maximum entropy change $\Delta S_{M}$ values calculated from the Maxwell equation ${ }^{35}$ at a constant temperature of $420 \mathrm{~K}$ is shown in Figure 2. As in the $\Delta T_{a d}$ measurements, an anisotropic behavior is observed in the field dependent $\Delta S_{M \text {,max }}$ measurements. Even in fields up to $4 \mathrm{~T}$ (much higher than the anisotropy field of approximately $2 \mathrm{~T}$ ) the entropy changes do not converge and the effect of anisotropy cannot be overcome. The inset of Figure 2 shows $\Delta S_{m}(T)$ for a field change of $2 \mathrm{~T}$ exhibiting a similar shape and peak temperature for both polycrystalline and single crystal samples. As in the case of the adiabatic temperature change measurements, the values of the polycrystalline sample lie in between the ones of the single crystal with fields applied in the easy plane and hard axis. Both the above described effects of $\Delta T_{a d}$ and $\Delta S$ that occur in the polycrystalline samples can be explained by an arbitrary grain orientation leading to values lying in between these values obtained for field alignment along the easy plane and hard axis of magnetization, respectively.

In order to quantitatively study the physical origin of this effect, the temperature dependence of anisotropy energy $E_{a}$ was calculated in different field changes up to $4 \mathrm{~T}$ in a temperature range between $360 \mathrm{~K}$ and $470 \mathrm{~K}$. The results are shown in Figure 3(a). $E_{a}$ was determined by subtracting the integration of $M-H$ at constant $T$ for different fields applied parallel to the [100] axis and (100) plane. $E_{a}$ is sketched as the filled area in the inset of Figure 3(a).

The values of magnetocrystalline anisotropy are rather low compared to other hard magnetic materials ${ }^{36}$ yet two things are rather peculiar: first, $E_{a}$ is still existent above $T_{C}$ and second, $E_{a}$ does not saturate with fields above the anisotropy field. This is obvious when looking at the recorded $M-H$ curves in the inset of Figure 3(a) where the magnetization versus field is plotted for the temperatures of

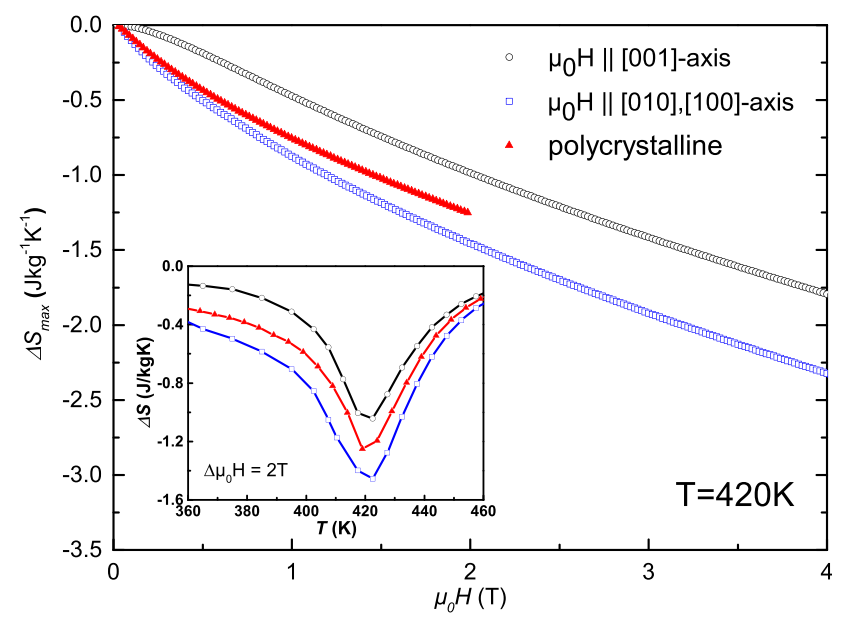

FIG. 2. Field dependence of $\Delta S_{\max }$ for fields applied parallel to [100] and [001] axes and to a polycrystalline sample. The inset displays the temperature dependence of $\Delta S_{m}$ in a field change of $2 \mathrm{~T}$. 

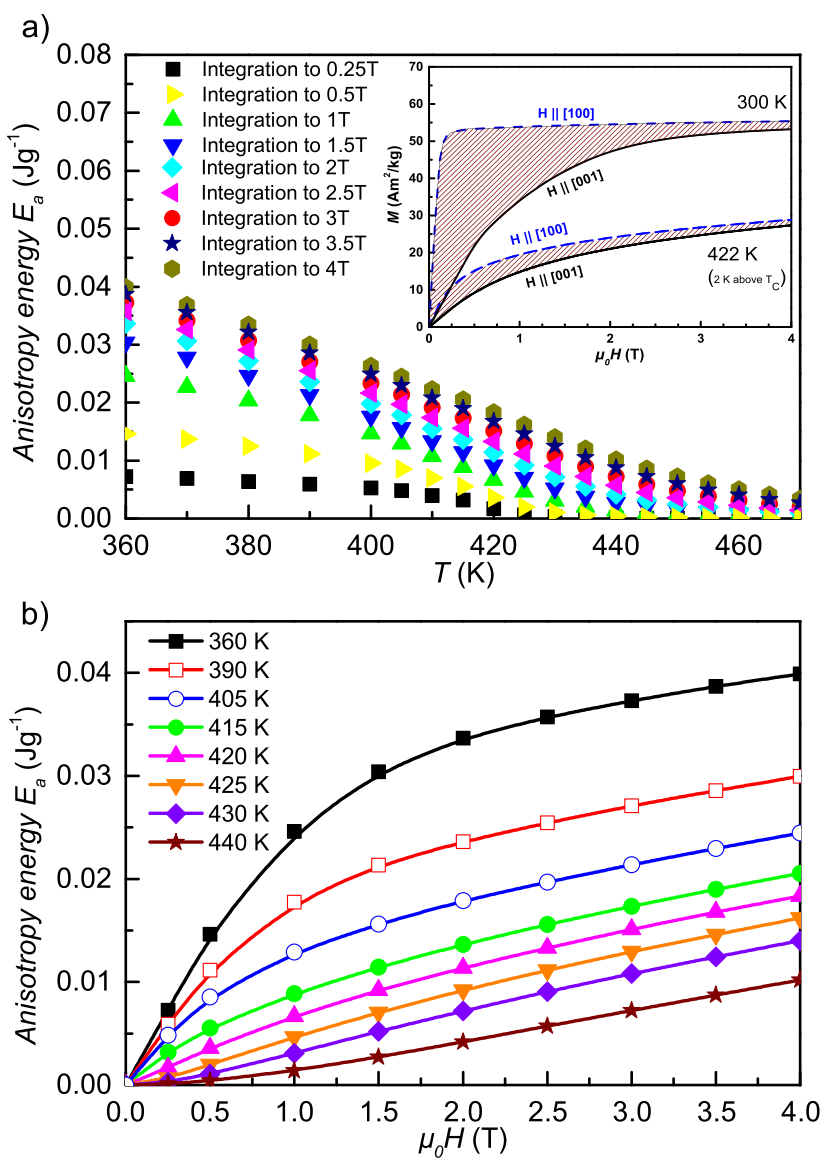

FIG. 3. (a) Anisotropy energy $\left(E_{a}\right)$ vs temperature for fields ranging from $0.25 \mathrm{~T}$ to $4 \mathrm{~T}$ for the $\mathrm{Co}_{2} \mathrm{~B}$ single crystal. The inset shows the field dependence of magnetization at two distinct temperatures. The hatched area indicates $E_{a}$. (b) Field dependence of $E_{a}$ at different temperatures below and above $T_{C}$.

$300 \mathrm{~K}$ and $422 \mathrm{~K}$ ( $2 \mathrm{~K}$ above $T_{C}$, determined by Arrott plots). It can be clearly seen that $M$ does not fully saturate, making the integral non-converging; furthermore, anisotropy energy (marked by the hatched area) is still existent above $T_{C}$ defying Zener's theory of temperature dependence of anisotropy ${ }^{37}$ This phenomenon becomes even more obvious when looking at Figure 3(b) where the field dependence of $E_{a}$ is plotted at temperatures above and below $T_{C}$. Above the Curie temperature of $420 \mathrm{~K}, E_{a}$ is still increasing almost linearly with field.

The effect of magnetization anisotropy was predicted by Callen and Callen ${ }^{38,39}$ and described as a alignment of magnetic moments within a narrow cone along the easy axis of magnetization and an alignment along a wider cone in the hard direction. This effect directly also correlates with a difference in paramagnetic susceptibilities.

To estimate the contribution of anisotropy energy on the magnitude of the anisotropic MCE, the rotational magnetocaloric effect was calculated. As proposed by Nikitin et al. in Ref. 26, the rotational entropy change $\Delta S_{\text {rot }}$ under adiabatic conditions can be calculated by the following expression:

$$
\Delta S_{r o t}=-\left[\left(\frac{\delta E_{a, H}}{\delta T}\right)-\left(\frac{\delta E_{a, 0}}{\delta T}\right)\right]=-\left(\frac{\delta \Delta E_{a}}{\delta T}\right),
$$

where $E_{a, H}$ and $E_{a, 0}$ are the magnetocrystalline anisotropy energy in the field $H$ and in the zero magnetic field, respectively. The results of the $\Delta S_{\text {rot }}$ calculations obtained by Equation (1) are shown in Figure 4. A peak in the rotational entropy change is found at $T_{C}$, coinciding with the measurements shown in Figure 2. In fields above $1 \mathrm{~T}$, the magnitude of the rotational MCE saturates. This is due to the fact that the differences in field dependent anisotropy energy are mostly only influenced by the anisotropic saturation magnetization, as can be seen in Figure 3.

Figure 5 shows the maximum values of $\Delta S_{\text {rot }}$ at a constant temperature of $420 \mathrm{~K}$ (at $T_{C}$ ). Additionally, the differences in entropy changes between the easy plane (100) and hard axis [001] calculated by the Maxwell equation $\left(\Delta S_{\max , 001}-\Delta S_{\max , 100}\right)$ are shown, which are directly correlated with the anisotropic magnetocaloric effect. The field dependence of entropy changes values obtained by both methods is similar, suggesting that the effect of the anisotropic magnetocaloric effect in the studied material system is due the anisotropy energy of the system.

The inset of Figure 5 shows a factor, we defined as the anisotropy factor, describing the potential loss of the magnetocaloric effect due to magnetocrystalline anisotropy as

$$
\begin{aligned}
\eta_{\text {anisotropy }} & =\frac{\left(\Delta S_{\max , \text { easy }}-\Delta S_{\max , \text { hard }}\right)}{\Delta S_{\max , \text { easy }}} \\
& =\frac{\left(\Delta S_{\max ,[001]}-\Delta S_{\max ,(100)}\right)}{\Delta S_{\max ,[001]}} .
\end{aligned}
$$

The dotted lines in the inset of Figure 5 indicate a potential loss in entropy change of $50 \%$ in a field change of $1 \mathrm{~T}$ and $35 \%$ in $1.9 \mathrm{~T}$. The difference in entropy changes between applying a field in the easy plane and hard axis of magnetization is less pronounced than in the case of adiabatic temperature changes (see Figure 1).

The field dependence of the anisotropy factor (Fig. 5) follows an exponential phenomenological decay law with

$$
\eta_{\text {anisotropy }}(H)=0.25+0.79 \exp \left(\frac{-H}{0.86}\right),
$$

suggesting a large influence of magnetocrystalline anisotropy energy especially in low fields below $1 \mathrm{~T}$. This finding is of special importance as this field range is aimed for in a real

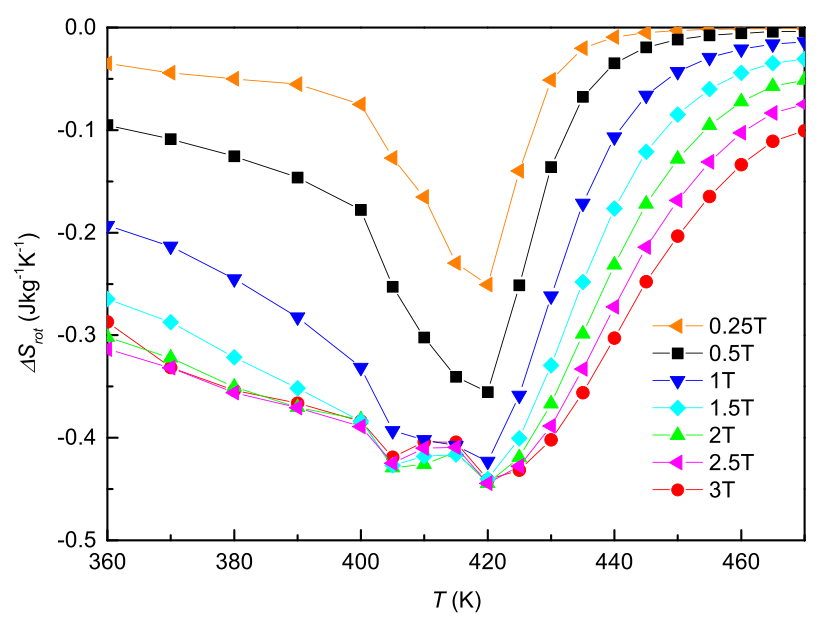

FIG. 4. Rotational entropy change $\Delta S_{\text {rot }}$ versus temperature plotted for different fields. 


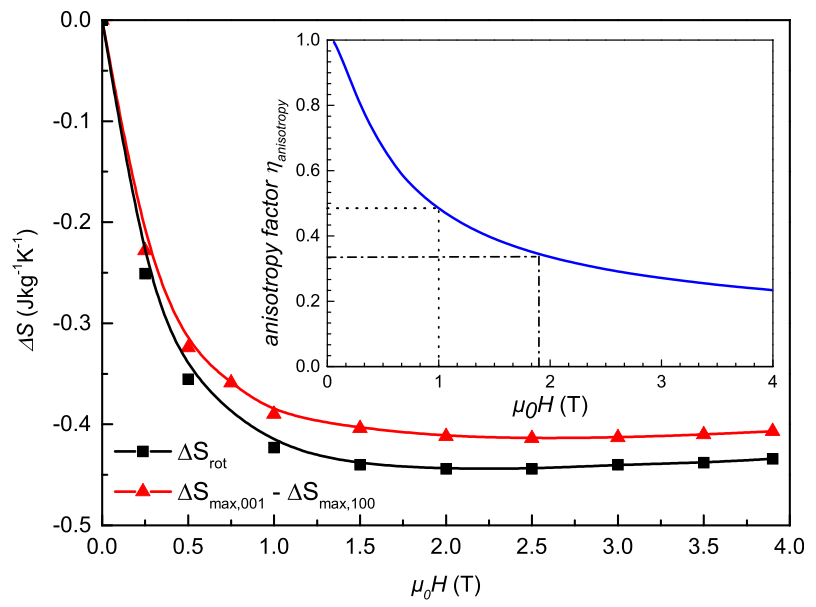

FIG. 5. The maximum values of $\Delta S_{\text {rot }}$ at constant temperature (black squares) and the difference of maximum entropy changes in easy and hard directions (red triangles). The inset shows the field dependence of the anisotropy factor described in Equation (2).

household refrigeration scenario as the permanent magnet setup will play the greatest role in price and eco-friendliness as described in Ref. 40. This finding shows that the magnetocaloric effect will be underestimated in measurements of the MCE on polycrystalline materials exhibiting magnetocrystalline anisotropy. Even more important, it shows that special care needs to be taken when producing heat exchangers for active magnetocaloric regenerators comprised of non-cubic magnetocaloric materials like for example, polymer bonded plates as shown in Ref. 41. In order to maximize the magnetocaloric effect and therefore make use of the magnetic field source as efficiently as possible, the particles should be aligned parallel to the applied magnetic field with respect to their easy axis of magnetization.

In conclusion, the majority of studies of the magnetocaloric effect (MCE) near $T_{C}$ are performed by using isotropic polycrystalline samples where the contribution coming from magnetocrystalline anisotropy is averaged out. Whereas in the case of cubic symmetry, the use of polycrystalline samples is justified, in the case of non-cubic magnetic materials we could show that the magnetic anisotropy near $T_{C}$ is important. MCE measurements on polycrystalline samples lead to underestimation of $\Delta T_{a d}$ and $\Delta S$. Especially in the target scenario of utilizing materials in small magnetic fields (e.g., 1 to $2 \mathrm{~T}$ ), the effect of anisotropy is of great importance. Furthermore, due to the nature of $\Delta S$, the effect of anisotropy cannot be overcome even in high fields available in the laboratory. We therefore propose utilizing textured non-cubic magnetocaloric materials with the easy axis of magnetization parallel to the applied field in a real magnetocaloric working device to increase the overall efficiency.

The research leading to these results has received funding from the European Community's Seventh Framework Programme FP7/2007-2013 under Grant Agreement No. 310748 (DRREAM). We thank the DFG (Grant No. SPP 1599) for financial support. V.F. acknowledges funding from the Deutsche Akademischer Austauschdienst DAAD Award
A/13/09434 and the Spanish MINECO and EU-FEDER (projects MAT2013-45165-P and MAT2016-77265-R). D. Yu. K. gratefully acknowledges the financial support of the framework of Increase Competitiveness Program of NUST MISiS (No. K4-2015-013). The support from the German federal state of Hessen through its excellence programme LOEWE“RESPONSE" is acknowledged. We thank C. A. Schwoebel for his help with the Laue Camera.

${ }^{1}$ C. Zimm, A. Jastrab, A. Sternberg, V. Pecharsky, K. Gschneidner, Jr., M. Osborne, and I. Anderson, "Description and performance of a near-room temperature magnetic refrigerator," in Advances in Cryogenic Engineering, Vol. 43, edited by P. Kittel (Springer US, 1998), Sect. 222, pp. 1759-1766.

${ }^{2}$ O. Gutfleisch, M. A. Willard, E. Brück, C. H. Chen, S. G. Sankar, and J. P. Liu, Adv. Mater. 23, 821 (2011).

${ }^{3}$ V. K. Pecharsky and J. K. A. Gschneidner, Phys. Rev. Lett. 78, 4494 (1997).

${ }^{4}$ F. Hu, B. Shen, J. Sun, Z. Cheng, G. Rao, and X. Zhang, Appl. Phys. Lett. 78, 3675 (2001).

${ }^{5}$ A. Fujita, S. Fujieda, Y. Hasegawa, and K. Fukamichi, Phys. Rev. B 67, 104416 (2003).

${ }^{6}$ J. Lyubina, R. Schäfer, N. Martin, L. Schultz, and O. Gutfleisch, Adv. Mater. 22, 3735 (2010).

${ }^{7}$ J. Liu, J. D. Moore, K. P. Skokov, M. Krautz, K. Löwe, A. Barcza, M. Katter, and O. Gutfleisch, Scr. Mater. 67, 584 (2012).

${ }^{8}$ O. Tegus, E. Brück, K. H. J. Buschow, and F. R. de Boer, Nature 415, 150 (2002).

${ }^{9}$ A. Yan, K. H. Müller, L. Schultz, and O. Gutfleisch, J. Appl. Phys. 99, 08K903 (2006).

${ }^{10}$ F. Guillou, G. Porcari, H. Yibole, N. van Dijk, and E. Brück, Adv. Mater. 26, 2671 (2014).

${ }^{11}$ J. Liu, T. Gottschall, K. P. Skokov, J. D. Moore, and O. Gutfleisch, Nat. Mater. 11, 620 (2012).

${ }^{12}$ T. Gottschall, K. P. Skokov, D. Benke, M. E. Gruner, and O. Gutfleisch, Phys. Rev. B 93, 184431 (2016).

${ }^{13}$ A. Chirkova, K. P. Skokov, L. Schultz, N. V. Baranov, O. Gutfleisch, and T. G. Woodcock, Acta Mater. 106, 15 (2016).

${ }^{14}$ X. Moya, S. Kar-Narayan, and N. D. Mathur, Nat. Mater. 13, 439 (2014).

${ }^{15}$ V. Franco, J. S. Blazquez, B. Ingale, and A. Conde, Annu. Rev. Mater. Res. 42, 305 (2012).

${ }^{16}$ O. Gutfleisch, T. Gottschall, M. Fries, D. Benke, I. Radulov, K. P. Skokov, H. Wende, M. Gruner, M. Acet, P. Entel, and M. Farle, Philos. Trans. R. Soc., A 374, 2015.0308 (2016).

${ }^{17}$ J. V. Leitão, M. van der Haar, A. Lefering, and E. Brück, J. Magn. Magn. Mater. 344, 49 (2013).

${ }^{18}$ L. Caron, M. Hudl, V. Höglin, N. H. Dung, C. P. Gomez, M. Sahlberg, E. Brück, Y. Andersson, and P. Nordblad, Phys. Rev. B 88, 094440 (2013).

${ }^{19}$ S. V. Vonsovsky, Sov. Phys. J. Exp. Theor. Phys. 8, 1104 (1938).

${ }^{20}$ N. S. Akulov and L. W. Kirensky, J. Phys. 3, 31 (1940).

${ }^{21}$ A. S. Andreenko, P. B. Konstantin, S. A. Nikitin, and M. T. Aleksandr, Sov. Phys. Uspekhi 32, 649 (1989).

${ }^{22}$ K. P. Skokov, Y. G. Pastushenkov, S. A. Nikitin, M. Fries, and O. Gutfleisch, IEEE Trans. Magn. 52, 1 (2016).

${ }^{23}$ L. H. Bennett, R. D. McMichael, R. D. Shull, L. J. Swartzendruber, and R. E. Watson, J. Appl. Phys. 73, 6507 (1993).

${ }^{24}$ M. Zou, Y. Mudryk, V. K. Pecharsky, K. A. Gschneidner, D. L. Schlagel, and T. A. Lograsso, Phys. Rev. B 75, 024418 (2007).

${ }^{25}$ X. X. Zhang, H. L. Wei, Z. Q. Zhang, and L. Zhang, Phys. Rev. Lett. 87, 157203 (2001).

${ }^{26}$ S. A. Nikitin, K. P. Skokov, Y. S. Koshkidko, Y. G. Pastushenkov, and T. I. Ivanova, Phys. Rev. Lett. 105, 137205 (2010).

${ }^{27}$ J.-L. Jin, X.-Q. Zhang, G.-K. Li, Z.-H. Cheng, L. Zheng, and Y. Lu, Phys. Rev. B 83, 184431 (2011).

${ }^{28}$ P. K. Das, A. Bhattacharyya, R. Kulkarni, S. K. Dhar, and A. Thamizhavel, Phys. Rev. B 89, 134418 (2014).

${ }^{29}$ M. Balli, S. Jandl, P. Fournier, and M. M. Gospodinov, Appl. Phys. Lett. 104, 232402 (2014).

${ }^{30}$ H. Zhang, Y. Li, E. Liu, Y. Ke, J. Jin, Y. Long, and B. Shen, Sci. Rep. 5, 11929 (2015).

${ }^{31}$ Y. Hu, Q. B. Hu, C. C. Wang, Q. Q. Cao, W. L. Gao, D. H. Wang, and Y. W. Du, Solid State Commun. 250, 45 (2017). 
${ }^{32}$ A. Edström, M. Werwiski, D. Iuan, J. Rusz, O. Eriksson, K. P. Skokov, I. A. Radulov, S. Ener, M. D. Kuz'min, J. Hong, M. Fries, D. Y. Karpenkov, O. Gutfleisch, P. Toson, and J. Fidler, Phys. Rev. B 92, 174413 (2015).

${ }^{33}$ K. P. Skokov, K. H. Müller, J. D. Moore, J. Liu, A. Y. Karpenkov, M. Krautz, and O. Gutfleisch, J. Alloys Compd. 552, 310 (2013).

${ }^{34}$ H. Kadomatsu, F. Ishii, and H. Fujiwara, J. Phys. Soc. Jpn. 47, 1078 (1979).

${ }^{35} \mathrm{~A}$. Tishin and Y. Spichkin, The Magnetocaloric Effect and its Applications, Condensed Matter Physics (CRC Press, 2016).
${ }^{36}$ M. D. Kuz'min, K. P. Skokov, H. Jian, I. Radulov, and O. Gutfleisch, J. Phys.: Condens. Matter 26, 064205 (2014).

${ }^{37}$ S. Chikazumi, Physics of Ferromagnetism, International Series of Monographs on Physics (OUP, Oxford, 2009).

${ }^{38}$ E. R. Callen, J. Appl. Phys. 31, S149 (1960).

${ }^{39}$ E. R. Callen and H. B. Callen, J. Phys. Chem. Solids 16, 310 (1960).

${ }^{40}$ B. Monfared, R. Furberg, and B. Palm, Int. J. Refrig. 42, 69 (2014).

${ }^{41}$ K. P. Skokov, D. Y. Karpenkov, M. D. Kuzmin, I. A. Radulov, T. Gottschall, B. Kaeswurm, M. Fries, and O. Gutfleisch, J. Appl. Phys. 115, 17A941 (2014). 